\title{
THE PATHOGENESIS OF ANTERIOR TIBIAL SYNDROME
}

\section{An Illustrative Case}

\author{
David F. Paton, London, England \\ From the Whittington Hospital, London
}

The anterior tibial syndrome is now a well recognised entity and much has been written of it since Horn (1945) described two cases of contracture of the tibialis anterior and long extensors of the toes occurring in young soldiers, which he attributed to damage to the anterior tibial artery from the repetitive minor trauma of strenuous exercise. Hughes (1948) and others have since described cases of acute ischaemia with necrosis of muscle in the anterior tibial compartment occurring under similar circumstances, and Mavor (1956) described a chronic form of this condition resembling intermittent claudication with changes confined to the anterior tibial muscles.

The pathogenesis of this lesion has evoked considerable controversy. Two main theories have arisen. It is proposed in this article to discuss both, and to present a case history which seems to support one of them.

\section{CASE REPORT}

A man aged thirty-four presented in July 1963 with a swelling over the front of the shin which had been present for six months. There was a vague history of injury five months before this, but the swelling had appeared gradually. On examination there was a swelling over the anterior tibial muscles in the middle third of the leg, three centimetres in diameter. There was no evidence at that time of contracture or loss of function of the muscles. A diagnosis of muscle hernia was made. At operation in January 1964 the fascial defect was repaired. No dissection of the muscles was made at the time; so no damage could have been done to the anterior tibial artery. No abnormality of the muscles was observed. The fascial defect was closed with chromic catgut sutures and the observation made that it was closed with "some degree of tension."

Three days after the operation the patient was pyrexial and the leg was very tender. He was treated for presumed wound infection. A week after operation cellulitis was present but there was no purulent discharge. Subsequently a persistent discharge of serous fluid occurred but repeated bacteriological cultures failed to grow any organism. After ten months he was readmitted. In addition to the persistent discharge there were weakness and aching in the ankle. Examination revealed a contracture of the anterior tibial muscles which prevented flexion of the ankle. There was a firm swelling beneath the operation scar, which was still discharging serous fluid. Radiographs showed considerable cortical thickening of the anterior border of the tibia (Fig. 1).

Fig. 1

Radiographs taken before operation showing the cortical thickening of the tibia.

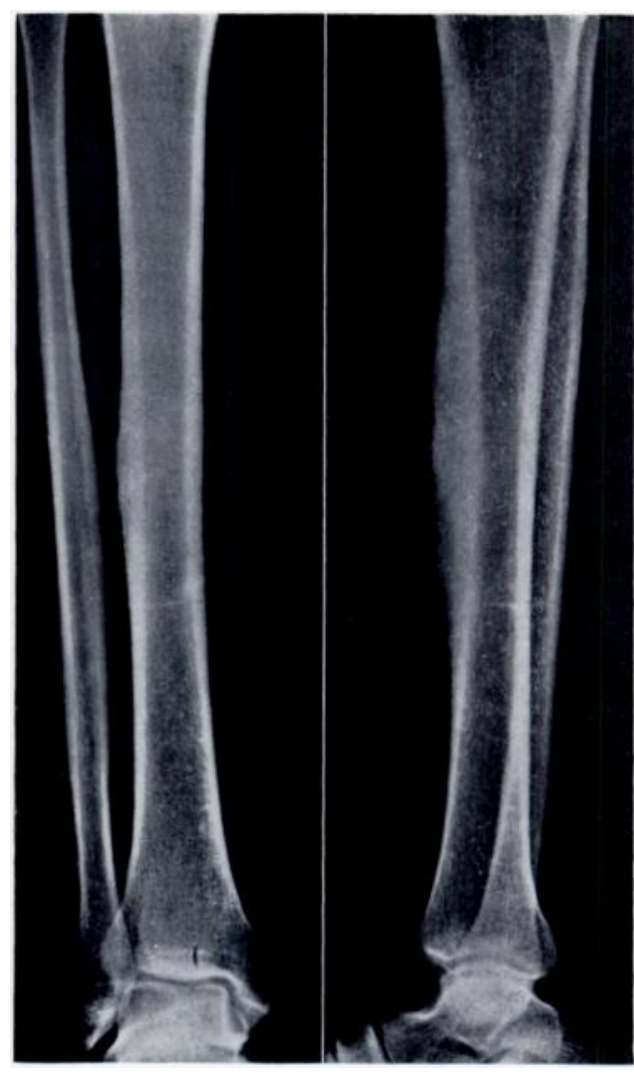

FIG. 1 
Operation-The anterior tibial compartment was explored. Division of the fascia revealed an area of the anterior tibial muscles, about four inches long, which was completely replaced by fibrous tissue. The tibialis anterior was the worst affected muscle. The centre of this area was replaced by necrotic xanthochromic putty-like material, which was presumed to be the source of the persistent discharge. There was thickening of the adjacent cortex of the tibia. The whole of the necrotic part of the tibialis anterior was resected. The limb was immobilised in plaster. Progress-The patient made an uneventful recovery. The wound healed without further discharge and apart from stiffness of the ankle the patient was free from symptoms when last seen five months later.

Histological examination of the specimen showed massive ischaemic necrosis of part of the tibialis anterior with severe chronic inflammatory infiltration in the surrounding muscle and tendon including many giant-cell forms in muscle fibres and some old blood pigmentation. Examination of a specimen of bone from the tibia showed cortical osteosclerosis with subperiosteal reactive new bone formation. Culture of the specimen revealed a few B. Proteus in anaerobic culture only and was reported " of doubtful significance."

\section{DISCUSSION}

Of the two theories concerning the pathogenesis of this ischaemic lesion, the first is that a vascular accident involves the anterior tibial artery. The arterial lesion has been variously ascribed to repeated injury, thrombosis or spasm. In no cases has primary disease of arteries been present.

Horn (1945) suggested that repeated overwhelming demands made upon the anterior tibial artery during strenuous exercise predisposed to fibrosis of the media, adventitia and periarterial tissue. He was uncertain what precipitated the subsequent acute episode but suggested that it might be caused by arterial spasm, thrombosis or increased tension in the anterior tibial compartment. However, he produced no evidence to deny the possibility that the reported histological changes in the artery might be secondary to the inflammatory changes in the adjacent muscle.

The possibility of arterial embolus as a cause was refuted by Hughes (1948), who pointed out that in none of the recorded cases was a source of embolus ever found, and that the right angle at which the anterior tibial artery leaves the popliteal trunk makes it an unlikely site for lodgement of an embolus.

Localised thrombosis of main limb arteries, occurring without apparent cause, was described by Learmonth, Blackwood and Richards (1944). But in none of the cases reviewed by Carter, Richards and Zachary (1949) had there been evidence of ischaemia of the lower leg as a whole, and in only one case has there been a recorded loss of the dorsalis pedis pulse. Hughes (1948) proved by injection studies that to produce severe ischaemia of the muscles in the anterior tibial compartment a long segment of the anterior tibial artery would have to be occluded. He even suggested that selective sparing of the anterior tibial recurrent artery might be responsible for the fact that the muscle in the upper part of the compartment is usually, but not invariably, spared. It seems unlikely that such a long segment of the artery could be excluded so selectively as to give no other evidence of a vascular accident. Hughes (1948) himself preferred the suggestion that arterial spasm of the long segment caused the ischaemia. He agreed, however, with Carter et al. (1949) that this spasm would have to persist for six to twenty-four hours to cause this irreversible lesion.

It seems most unlikely that such selective spasm, occurring so rarely, and lasting for so long without producing detectable thrombosis of the artery, could be the cause of this condition. It seems also unlikely that this severe spasm could follow the minor trauma of heavy exercise.

The alternative theory of the pathogenesis of the anterior tibial syndrome is that increased tension in the anterior tibial compartment embarrasses the blood supply of the muscles. It is a well known fact that the anterior tibial compartment is a narrow space with rigid walls. 
The muscles, nerves and vessels run along this space, and are thus vulnerable should there be a rise in tension. It has been suggested that sudden severe exercise, particularly in untrained men, causes swelling of muscle and a rise in tension in the compartment which embarrasses the circulation. Wright (1952) showed that after prolonged exercise a muscle may retain so much fluid as to increase its weight by 20 per cent and hence its bulk. Carter et al. (1949) stated that they believed this to be the cause of the rise in tension, but added that rupture of muscle fibres and haemorrhage into the muscles were contributory factors.

Hughes (1948) criticised this theory on the grounds that the anterior tibial compartment is not like a closed box but is open inferiorly. Mavor (1956), however, felt that this opening is only a potential space as it is plugged with tendons. Hughes also made the observation, confirmed by others, that the tibialis anterior and extensor hallucis longus muscles are involved more severely than the extensor digitorum longus, this observation being confirmed in the case here presented. He objected to the concept of a universal rise in tension which occludes some blood vessels and not others, observing that pressures great enough to occlude the main vessels would occlude all the intramuscular vessels in the compartment.

Lewis (1936) observed that much lower pressures would occlude the fine intramuscular vessels without occluding the main vessels-only 50 to 60 millimetres of mercury was sufficient. This fact seems to allow of the possibility of localised areas of ischaemia in muscle dictated by local anatomical dimensions.

Mavor (1956) described a case of chronic and intermittent ischaemia of the anterior tibial muscles of both legs in a young footballer. The symptoms were completely relieved by division of the anterior crural fascia. He noted as evidence of the presence of increased tension in the compartment the fact that the patient had muscle herniae over the anterior tibial muscles of both legs. The case presented here also had this feature.

There are now two cases recorded in which ischaemic necrosis of muscle has followed the artificial raising of pressure by surgical intervention. In 1944 Sirbu, Murphy and White reported a case in which muscle necrosis followed the closure of a muscle hernia through the anterior crural fascia. On the fifth day after operation fever and local signs of inflammation appeared. Sutures were removed to reveal greyish-white gangrene of the anterior tibial muscles. The case reported here is the second case. Here a muscle hernia was closed with the same result, and it is known that no dissection near the anterior tibial artery was performed.

\section{SUMMARY}

1. A case of hernia of the anterior tibial muscles is described in which repair of the hernia under tension was followed by local muscle necrosis and contracture.

2. This case supports the theory of pathogenesis of the anterior tibial syndrome that increased tension in the compartment embarrasses the blood supply of the contained muscles.

I wish to thank Mr Ian Winchester for his permission to publish this case.

\section{REFERENCES}

Carter, A. B., Richards, R. L., and Zachary, R. B. (1949): The Anterior Tibial Syndrome. Lancet, ii, 928. HoRn, C. E. (1945): Acute Ischaemia of the Anterior Tibial Muscle and the Long Extensor Muscles of the Toes. Journal of Bone and Joint Surgery, 27, 615.

Hughes, J. R. (1948): Ischaemic Necrosis of the Anterior Tibial Muscles due to Fatigue. Journal of Bone and Joint Surgery, 30-B, 581.

LeARMONTH, J. R., BlaCKWOOD, W., and Richards, R. L. (1944): Localised Arterial Thrombosis of Indeterminate Origin. Edinburgh Medical Journal, 51, 1.

LEWIS, T. (1936): Vascular Disorders of the Limbs, p. 36. London: Macmillan \& Co. Limited.

MAvor, G. E. (1956): The Anterior Tibial Syndrome. Journal of Bone and Joint Surgery, 38-B, 513.

Sirbu, A. B., MUrPhy, M. J., and White, A. S. (1944): Soft Tissue Complications of Fractures of the Leg. California and Western Medicine, 60, 53.

Wright, S. (1952): Applied Physiology: Ninth edition, p. 19. London: Oxford University Press.

VOL. 50 B, NO. 2, M.AY 1968 